\title{
De novo mutations in GFAP cause Alexander disease: clinical features, fMRI and functional analysis
}

\author{
Xiaoxuan Song ${ }^{1}$, Jingwen Jiang ${ }^{1}$, Wotu Tian $^{1}$, Feixia Zhan ${ }^{1}$, Zeyu Zhu ${ }^{1}$, Xiaojun Huang${ }^{1}$, \\ Xiaoli $\mathrm{Liu}^{2}$, Binyin $\mathrm{Li}^{1}$, Huidong Tang${ }^{1}$, and $\mathrm{Li} \mathrm{Cao}^{3}$ \\ ${ }^{1}$ Shanghai Jiao Tong University Medical School Affiliated Ruijin Hospital \\ ${ }^{2}$ Shanghai Fengxian Central Hospital \\ ${ }^{3}$ Rui Jin Hospital affiliated to Shanghai Jiao Tong University School of Medicine
}

August 3, 2020

\begin{abstract}
Two de novo mutations in GFAP gene were identified (c.214G > A, p.E72K and c.1235C > T, p.T412I) by whole exome sequencing. The common clinical features of the two patients was bulbar dysfunction, pyramidal signs and white matter lesions in periventricular regions. We conducted a novel data-driven method to explore the atrophic pattens and spontaneous brain functional network activity according to the neuroimaging data. Similar atrophic patterns, increased brain functional connectivity in occipital and posterior parietal cortex were detected in the two probands. Western blotting revealed the decreased level of GFAP with p.T412I mutation, while p.E72K and p.R239C mutations were at a similar level to wild type, suggesting the mutations located in the tail domain could decrease the solubility of GFAP. Abnormal inclusions of mutant GFAP were colocalized with ubiquitin, 20S proteasome, protein 1 light chain 3-II (LC3-II) and lysosome. The mutant GFAP caused activated autophagy flux while ubiquitin-proteasome pathway could be blocked as a mechanism for degrading aggregates. We herein presented two AxD patients with heterozygous mutations in GFAP. We noticed that mutant GFAP aggregations induced activated autophagy upon proteasome degrading pathway impairment. Our findings further expand the clinical and genetic spectrum of AxD.
\end{abstract}

\section{Introduction}

Alexander disease $(\mathrm{AxD})$ is a progressive and fatal neurological disorder characterized by white matter degeneration caused by heterozygous mutations in the GFAP gene, which encodes the intermediate filament protein in astrocytes. The pathological hallmark of $\mathrm{AxD}$ is the presence of astrocytic cytoplasmic inclusions named Rosenthal fibers (RFs). These inclusions contain glial fibrillary acidic protein (GFAP), small heat shock proteins hsp27 and $\alpha \mathrm{B}$-crystallin. In 2001, Messing et al. identified GFAP as a candidate gene for AxD (Brenner et al., 2001). Since then, more than 550 cases have been reported, most of them were missense mutations ("Alexander's Disease,"). AxD has been classified into three types proposed by Yoshida based on the neurological and MRI findings: cerebral form (type 1), bulbospinal form (type 2) and intermediate form (type 3). Type $1 \mathrm{AxD}$ is characterized by delayed psychomotor development, convulsions, macrocephaly and leukoencephalopathy with frontal lobe predominance on brain imaging. Type 2 patients with atrophy of medulla oblongata or cervical cord on MRI generally showed muscle weakness, hyperreflexia and distinct bulbar dysfunction. Type 3 is an intermediate form which contains various symptoms of type 1 and type 2 (Yoshida et al., 2011). In addition to the featured clinical manifestations and brain MRI imagings, the definitive diagnosis for AxD still requires genetic analysis or brain biopsy.

The pathogenic mechanisms mediating AxD remain undefined. Previous studies suggested that mutant GFAP could implicate intracellular vesicle regulation and organelle distribution, leading to abnormal accumulations of eosinophilic cytoplasmic inclusions and dysfunctional consequences (Jones et al., 2018). 
Aggregate-prone proteins unsuccessfully corrected by chaperones are likely to be ubiquitylated and subsequently recognized by protein degrading pathways, such as the ubiquitin-proteasome system (UPS) and the autophagy-lysosomal pathway (ALP) (Kwon \& Ciechanover, 2017). However, mutant GFAP can inhibit proteasome activity and result in elevated levels of ubiquitinated proteins. The proteasome impairment in turn activates intracellular stress response, resulting in alteration of protein levels caused by up-regulated transcription and activated autophagy.

Here, we identified two AxD patients with de novo mutations in GFAP gene. Based on detailed clinical, neuroimaging and genetic analysis, functional investigations were further conducted to explore the pathogenicity of identified variants.

\section{Materials and Methods}

\section{Participants}

Two probands (P3433 and P4288) identified with AxD were enrolled. This study had received ethics approval from the Ethics Committee of Ruijin Hospital, Shanghai Jiao Tong University School of Medicine, Shanghai, China. All participants had signed informed consents.

\section{Genetical analysis}

Genomic DNA was extracted by phenol-chloroform method. 200 healthy subjects were enrolled as normal controls. Whole exome sequencing was performed for 2 probands. The human genome reference sequence (GRCh37/hg19) were used to map the sequence reads. All the GFAP variants were denoted as RefSeq NM_002055.5. On the basis of public databases of normal human variation (1000g, ExAC and gnomAD), all the variants of which Minor Allele Frequency (MAF) is higher than $1 \%$ were filtered. Polyphen-2 (http://genetics.bwh.harvard.edu/pph2), SIFT (http://provean.jcvi.org) and Mutationtaster (http://www.mutationtaster.org) were used to predict the pathogenicity of mutation. American College of Medical Genetics and Genomics (ACMG) Guidelines were used to interpret and classify variants. Sanger sequencing was carried out to confirm the putatively pathogenic variants.

\section{MRI acquisition, preprocessing and statistical analysis}

Two probands were scanned on a magnetic resonance system (Ingenia, Philips MR system, Dutch) with an 8-channel based-array head coil, with 15 normal individuals (5 male, 10 female participants) as controls. The protocol included a 3-dimension high-resolution turbo field echo T1-weighted sequence for neuroanatomy ( sagittal slice orientation; matrix $=256 \times 256$; repetition time $=7.2 \mathrm{~ms}$; echo time $=3.3 \mathrm{~ms}$; flip angle $=7^{\circ}$; slice thickness $=1 \mathrm{~mm}$; slice number $=192)$. Resting-state blood oxygen level dependent $($ BOLD) MRI used T2*-weighted echo planar imaging sequence (240 functional images, sagittal slice orientation; 39 slices; slice thickness $=3.5 \mathrm{~mm}$; matrix $=64 \times 64 ;$ repetition time $=2000 \mathrm{~ms}$; echo time $=30 \mathrm{~ms} ;$ flip angle $\left.=90^{\circ}\right)$. The two patients also had T2-weighted fluid attenuated inversion recovery (FLAIR) sequence to recognize white matter lesions better.

The T1-weighted anatomical image was firstly segmented into grey matter (GM), white matter (WM) and cerebrospinal fluid using computational anatomy toolbox (CAT) 12 (http://www.neuro.unijena.de) within SPM 12 (v6685; http://fil.ion.ucl.ac.uk/spm) in MATLAB 2014b environment (https://www.mathworks.com), with reference to tissue probabilistic maps in MNI (Montreal Neurological Institute) space. WM hyperintensity was also estimated by GM-WM tissue probability map in CAT12. Voxel based morphology analysis was performed between each patient and normal controls by test for GM and WM respectively, with total intracranial volume (TIV) as covariate. For multiple comparison correction, we used false discovery rate (FDR) correction at $\mathrm{p}<0.05$.

Regarding the fMRI, the first $40 \mathrm{fMRI}$ images of each subject were discarded. The remaining 200 images were realigned to adjust head motion, co-registered to the anatomical image and normalized into MNI space using a modified MATLAB toolbox "Data Processing \& Analysis of Brain Imaging (DPABI, version 3.0)" (Yan, Wang, Zuo, \& Zang, 2016). We computed mean amplitude of low frequency fluctuation (ALFF) to represent 
individuals' regional neural activities (Yang et al., 2007), regional homogeneity (Reho) and degree centrality (DC) representing the quantity of functional connections of a region (Eijlers et al., 2017; Zang, Jiang, Lu, He, \& Tian, 2004). Individual participants' weighted DC values were from for all voxels in standard space with DPABI. Mean ALFF and voxel-wise centrality values were also compared between one patient and controls with $t$ test adopting FDR of $\mathrm{p}<0.05$, with voxel-based morphometry of GM as covariate volume.

\section{Cell culture and transfection}

The cDNAs for identified variants in GFAP (NM_002055.5) were inserted into the pcDNA3.1-GFP plasmids to express GFP-tagged fusion proteins. HEK 293T cells were grown in Dulbecco's modified Eagle's medium supplement with $10 \%$ Fetal bovine serum (FBS) (Gibco) and $1 \%$ penicillin streptomycin (P/S) (Invitrogen). $24 \mathrm{~h}$ after plating, cells were transfected with GFAP-GFP wild-type (GFAP-WT) or mutant (p.E72K and p.T412I) plasmid DNA using Lipofectamine 3000 transfection reagent (Invitrogen). Moreover, a hot spot mutation (c.715C > T, p.R239C) (Li et al., 2005) identified as disease-causing was set as positive control. 24h after transfection, cells were collected to extract proteins for western blot. As for pharmacological treatment, MG132 (proteasome inhibitor) (10 uM) (Tang, Perng, Wilk, Quinlan, \& Goldman, 2010) (Cell Signaling Technology) and Bafilomycin AI (BafAI, lysosomal inhibitor) (5nM) (Bresciani et al., 2018) (Sigma) were added to post-transfected cells respectively and incubated for $12 \mathrm{~h}$ to extract proteins, with DMSO as the vehicle control.

\section{Western blotting and immunofluorescence}

Cells were collected and lysed in radioactive immunoprecipitation assay (RIPA) buffer (Beyotime) with protease inhibitors for protein extraction. After centrifuged at $13000 \mathrm{~g}$ for $20 \mathrm{~min}$ under 4 , cell lysates were separated into two parts, the supernatant as the soluble fraction, and the sedimentation as the "insoluble" fraction. The insoluble fraction was dissolved by denaturing protein solubilization reagent (Invent). Equal amounts of protein were diluted in 6X SDS-PAGE Sample Loading Buffer (Beyotime) and were then separated by $12 \%$ SDS-PAGE. The protein levels of EGFP-GFAP were detected by anti-GFP antibody (1:2500, AVES). Anti-proteasome 20S (1:2000, Enzo Life Science) and anti-ubiquitin antibodies (1:1000, Cell Signaling Technology) were used for the analysis of the UPS. Anti-LC3B (1:1000, Cell Signaling Technology) and anti-LAMP1 antibodies (1:1000, Cell Signaling Technology) were used to detect the ALP. We chose 20S and LC3 to undergo western blot, respectively. Using anti-GAPDH antibody (1:1000, Cell Signaling Technology) to normalize sample loading and transfer. Then the blots were incubated with secondary HRP-conjugated antibodies (1:5000, Beyotime) and subjected to chemiluminescence detection.

For immunofluorescence, $48 \mathrm{~h}$ after transfection, cells were fixed with $4 \%$ PFA, blocked with $10 \%$ serum and $0.3 \%$ Triton X-100 in PBS for 60min, and incubated with primary antibodies to detect the UPS and the ALP mentioned above in blocking solution at $4 \mathrm{deg} \mathrm{C}$ overnight. Then cells were stained with the Alexa Fluor 594 secondary antibodies (1:1000, Life), and nucleic acid was stained with DAPI (1:10000, Life). Cells were visualized under a Zeiss 710 confocal microscope.

\section{Clinical findings}

Patient P3433 was a 29-year-old woman featured as gait disturbance with a 19-year-duration. She had no issues of developmental retardation or psychomotor abnormalities. She was diagnosed with scoliosis (Fig. 1A) at the age of 14. Then she received corrective surgery for scoliosis (Fig. 1B) due to progressive walking difficulties. Likewise, her brain imaging showed mild leukoencephalopathy. There was no consanguineous marriage in her family. Neurological examination revealed strabismus, extraocular muscle movement disorder, several beats of bilaterally horizontal nystagmus and cerebellar ataxia. Muscle tension and strength of lower extremities were significantly decreased. The bilaterally pathologic signs were positive. She showed dysmetria in the finger-to-nose test on both sides. Her sensory system and cognitive functions were normal. Laboratory examinations revealed unremarkable findings. A de novo missense mutation in GFAP gene (NM_002055.5), c.214G >A (p.E72K) (Fig. 1C), which had been reported was identified in our study (Prust et al., 2011). This variant was absent in 1000 Genome Project (1000g, http://browser.1000genomes.org), Exome Aggregation Consortium (ExAC), gnomAD (http://gnomad-old.broadinstitute.org) and our controls. 
p.E72K was predicted damaging by PolyPhen2 (probability score 0.945), damaging by SIFT (score: 0.001) and disease-causing by Mutationtaster (probability score 1.000). According to the ACMG Guidelines, this variant was classified as likely pathogenic.

Patient P4288 was a 33-year-old man suffering slurred speech and progressive unsteady gait for two-year duration. Imaging examinations revealed thoracic spinal cord thinning and tethered cord syndrome. Surgical decompression of cauda equina nerve was performed and symptoms were slightly improved after operation. About one year later, he presented with swaying while walking caused by progressive spasticity and muscle weakness. Physical examinations revealed speech disfluency and horizontal nystagmus. Muscle strength in lower limbs were decreased. Pathologic reflexes of both sides were positive. Examinations of coordinate movement were difficult to complete. There was no significant abnormality in cognition and mental states. Laboratory investigations were nearly normal. A rarely reported variant(NM_002055.5), c.1235C $>$ T (p.T412I) (Fig. 1D), were detected in patient P4288 which was absent in 1000g, ExAC and gnomAD, as well as controls. SIFT (score 0.001), Polyphen2 (score 0.519) and mutationtaster (probability score 1.000) predicted the variants were damaging, possibly damaging and disease-causing, respectively. According to ACMG Guidelines, it was classified as likely pathogenic variant.

\section{Neuroimaging findings}

The brain MR imaging of the two probands displayed WM lesions in the bilateral corona radiata, centrum semiovale and the regions surrounding the 4 th ventricle (Fig. 1E to F). Volume estimation based on WM tissue probability map in CAT12 suggested $17.33 \mathrm{ml}$ of WM hyperintensity in patient P3433, while the TIV was $1218.15 \mathrm{ml}$. Comparatively, minor WM lesions were found in patient P4288.

The ages of control group ranged from 23 to 45 years old, with an average of 30.4 and standard deviation of 7.5. The averaged TIV was $1481.34 \mathrm{ml}$, with standard deviation of $113.83 \mathrm{ml}$. After adjusting for the TIVs, patient P3433 had atrophy mainly in the bilateral putamen and thalamus in the GM analysis. Consistent with leukodystrophies in the Alexander disease, atrophic WM was observed in the corona radiata, centrum semiovale, cerebellopontine angle and medulla. Patient P4288 presented similar pattern of atrophy in WM, while no significant GM difference was observed (Fig. 1G to $\mathrm{H}$ ).

Regarding the neural activity, higher ALFF in cerebellar vermis, cerebellopontine angles, occipital and posterior parietal cortex was observed in patient P3433, overlapping with higher DC and Reho in cerebellum and occipital cortex. Increased DC distributed in both frontal and posterior parietal cortex. In P4288, higher ALFF, DC and Reho was overlapped in similar regions. Increased DC was additionally observed in the bilateral insula (Fig. 1I to J).

\section{Variants functional analysis}

We evaluate the level of GFAP-WT/Mut proteins by western blotting, and the result showed the soluble GFAP of p.T412I group was lower than the WT group; Correspondingly, c.1235G > T(p.T412I) group exhibited a relatively higher level in the insoluble fraction. There was no significant difference among WT, c.214G $>$ A (p.E72K) and positive control (c.715C > T, p.R239C) in either soluble or insoluble fractions (Fig. 2A). By immunostaining, the WT exhibited diffuse distribution with a few aggregates of GFAP proteins throughout cytoplasm, while mutant GFAP (c.214G >A, c.1235G $>$ T and positive control) appeared as punctate aggregations in perinuclear areas (Fig. 2B).

Treated with proteasome inhibitor (MG132), GFAP proteins trended to decline especially in soluble WT, c.214G $>$ A(p.E72K )and positive control groups, while the $20 \mathrm{~S}$ proteasome complexes remained unchanged among all groups (Fig. 2C). Meanwhile, mutant GFAP were completely colocalized with ubiquitin and 20S, while in WT group, these proteins showed diffuse distribution in cytoplasm with no colocalization (Fig. 2D to E). We also tested the autophagy flux by the application of lysosome inhibitor (BafAI). The increased level of LC3-II was observed in soluble mutant groups when treated with inhibitor, and the overexpression of GFAP-WT could partly contribute to autophagy (Fig. 2F). Furthermore, the soluble GFAP level of mutant groups showed an upregulated tendency after BafAI treated, especially in the c.1235G $>$ T(p.T412I) 
and positive control group (Fig. 2G). By immunofluorescence, mutant GFAP nearly co-expressed with LC3 and lysosome (labeled by LAMP1) (Fig. 2H to I).

\section{Discussion}

Before our study, a total of 135 mutations in GFAP gene (NM_002055.5) have been reported, including 122 missense mutations, 1 splicing, 2 regulatory, 3 small deletion, 5 small insertion and 3 small indel mutations(Fig. 3) according to the Human Gene Mutation Database. The GFAP protein comprises an N-terminal head domain, a central rod domain and a C-terminal tail domain, which are important for assembling cellular intermediate filament (diameter, 10nm) (Hol \& Capetanaki, 2017). The rod domain manifesting a lower conservation in intermediate filaments contains four $\alpha$-helical segments (1A, 1B, 2A and 2B) (Li et al., 2005). Pathogenic mutations were scattered all over the domains of GFAP protein. However, the genotypephenotype correlations appear to be complicated. The variants affecting the hot spot amino acids (R79, R88, R239, R416) accounted for more than half of all mutations identified in AxD patients (Prust et al., 2011). The R79, R88 and R239 mutations are common in early-onset patients (Yoshida et al., 2011), while R239 mutations could induce more severe manifestations (Prust et al., 2011). Moreover, there exists a great deal of variability in clinical severity of $\mathrm{AxD}$, even among the individuals carrying the same mutation. Patient P3433 with c. $214 \mathrm{G}>\mathrm{A}$ (p.E72K) mutation which is located near R79, showed various symptoms of adult-onset form with relatively early-onset, such as bulbar symptoms, autonomic dysfunction and gait disturbance. Patient $\mathrm{P} 4288$ with c.1235C $>\mathrm{T}$ (p.T412I) variant which is located close to R416, presented a rapidly progressive clinical course. The patients with R416 mutation showed no obvious genotype-phenotype associations, supporting that mutations in tail domain of GFAP exhibit varied clinical courses and severities (Li et al., 2005). What accounts for these variabilities remains obscure, and the genetic modifiers or environmental impactors were considered as the possible factors affecting clinical phenotypes (Messing, 2018).

A novel data-driven method was used in our study to explore the atrophic pattern and spontaneous brain functional network activity in AxD patients. Patient P3433 and P4288 showed a similar pattern of atrophy in WM mainly involving periventricular regions. It has been proposed that the subventricular region was most vulnerable to the pathogenesis of AxD (Sawaishi, 2009). About one-third of the patients displayed abnormal signals in the periventricular rim (Balbi et al., 2010) which may be associated with the abnormal aggregation of Rosenthal fibers in subependymal regions (van der Knaap et al., 2006). Moreover, patient P3433 with early age onset also presented GM atrophy mainly in the bilateral putamen and thalamus, which has not been reported in AxD. GM volume loss may be related to long-term disability (A. Eshaghi et al., 2018) and interprets physical disabilities better than WM lesions (Roosendaal et al., 2011). Several mechanisms may underlie the GM damage including iron deposition, mitochondrial failure, WM lesions induced retrograde degeneration and meningeal inflammation (Calabrese et al., 2015; Arman Eshaghi et al., 2018). Patient P3433 suffered more severe WM atrophy, thus the overload and collapse of brain network could also explain the atrophy of GM (Minagar et al., 2013). For the first time, we evaluated alterations in spontaneous brain functional network activities in our patients by three different types of data-driven analyses, ReHo, DC and ALFF. The results showed the ReHo, DC and ALFF values seem to be consistently increased in occipital and posterior parietal cortex, indicating a higher amount of communication between these regions and the rest of the brain network. Increased functional connectivity has been interpreted as a compensatory mechanism or reorganization of the brain work (Widjaja, Zamyadi, Raybaud, Snead, \& Smith, 2013).

It is notable that the variant c.1235C $>\mathrm{T}$ (p.T412I) induced abnormal aggregations and significantly decreased solubility of GFAP, similar to the consequences caused by variant c.1178G $>$ T(p.S393I) (Chen, Lim, Chen, Quinlan, \& Perng, 2011) and c.1246C $>$ T(p.R416W) (Perng et al., 2006). The mutation with p.T412I, p.S393I and p.R416W are located in tail domain which is highly conserved and important for stabilizing filament-filament interactions (Yoshida, Sasayama, \& Nakagawa, 2009). Filament disorganizations may enhance the stability of the assembled protein, which could result in resistance to salt extraction (Hsiao et al., 2005). HEK 293T cells contain endogenous intermediate filaments such as vimentin and keratin which may have an effect on the assembly of filaments and GFAP network formation (Hsiao et al., 2005), but more research was needed to confirm. We likewise observed $20 \mathrm{~S}$ complexes colocalized with ubiquitylated 
GFAP inclusions, indicating the mutations did not disrupt GFAP binding to 20S complexes. Our results also exhibited the degradation of GFAP through the UPS seemed to be blocked. Previous studies demonstrated that overexpression of GFAP impairs proteasome activities and results in decreased protein turnover (Tang, $\mathrm{Xu}$, \& Goldman, 2006), especially mutant oligomer GFAP proteins produce a stronger inhibition (Tang et al., 2010). The loss of proteasome activity could be caused by the shift of $20 \mathrm{~S}$ complexes from soluble fraction to insoluble fraction (Tang et al., 2010). Proteasome dysfunction may establish a positive feedback loop which would further increase the GFAP protein levels and accumulations (Tang et al., 2006). However, some studies showed proteasome inhibitors could induce the reduction of GFAP gene transcription and other intermediate filaments expression (Middeldorp et al., 2009), as we observed the decreased GFAP levels in HEK 293T cells. This effect may only occur during the early stage (not after 7 days) of GFAP expression which is more susceptible to proteasome inhibition (Tang et al., 2010), while the relationship between proteasome inhibition and the expression of GFAP still needs explorations. As we had seen, the degradation of GFAP mutants could be restored by lysosome inhibitor, suggesting autophagy may act as a complementary pathway for degrading aggregated proteins in this disease (Tang et al., 2008). The GFP-tags and overexpression of GFAP plasmid may increase the formation of GFAP accumulations and exacerbate the pathological damage (Tang et al., 2006), which increased the difficulty to explore the mechanisms caused by GFAP mutants. It remains to be undefined whether other compensatory mechanisms exist for regulating the damaged proteins.

To summarize, we identified two cases of adult-onset $\mathrm{AxD}$ patients presenting with bulbar dysfunction and pyramidal tract signs. Two de novo variants (c.214G >A, p.E72K and c.1235C > T, p.T412I) in GFAP gene were identified, and the variant with c. $1235 \mathrm{C}>\mathrm{T}$ was explored the pathogenicity for the first time. Increased brain functional connectivity in occipital and posterior parietal cortex was discovered in two probands, while grey matter atrophy mainly in the bilateral putamen and thalamus was observed in the patient with more severe white matter damage. Our study broadened the spectrum of clinical phenotypes of AxD.GFAP mutations lead to abnormal accumulations and activate autophagy upon proteasome impairment to maintain intracellular homeostasis.

\section{Abbreviations}

AxD: Alexander disease; GFAP: glial fibrillary acidic protein; UPS: ubiquitin-proteasome system; ALP: autophagy-lysosomal pathway; GM: grey matter; WM: white matter; CAT: computational anatomy toolbox; TIV: total intracranial volume; DPABI: Data Processing \& Analysis of Brain Imaging; MNI: Montreal Neurological Institute; ALFF: amplitude of low frequency fluctuation; Reho: regional homogeneity; DC: degree centrality; FDR: false discovery rate; WT: wild-type; Mut: mutant; BafAI: Bafilomycin A1

\section{Web resources}

1000g: http://browser.1000genomes. org

EXAC or gnomAD: https://gnomad.broadinstitute.org/

Polyphen-2: http://genetics.bwh.harvard.edu/pph2

SIFT: http://provean.jcvi.org

Mutationtaster: http://www.mutationtaster.org

ACMG: https://www.acmg.net/

computational anatomy toolbox (CAT) 12: http://www.neuro.uni-jena.de

SPM 12: http://fil.ion.ucl.ac.uk/spm

MATLAB 2014b environment: https://www.mathworks.com

Human Gene Mutation Database: http://www.hgmd.cf.ac.uk/ac/index.php

\section{Acknowledgement}


The authors acknowledge the physicians and patients for their cooperation. This study was supported by the grants from the National Natural Science Foundation of China (No. 81870889 and No. 81571086), National Key R\&D Program of China (No. 2017YFC1310200 and No.2016YFC1305804), Shanghai Municipal Education Commission-Gaofeng Clinical Medicine Grant Support (No.20161401), Interdisciplinary Project of Shanghai Jiao Tong University (No. YG2016MS64).

\section{Authors' contributions}

XXS and JWJ were responsible for data acquisition, analysis and interpretation of data, study design, statistical analysis, drafting the manuscript. WTT, FXZ, ZYZ, XJH and XLL contributed to data acquisition, analysis and interpretation of data. BYL, HDT and LC designed this study and revised the manuscript. All authors read and approved the final manuscript.

\section{Availability of data and material}

All the data used and analyzed for this study are available from the corresponding author on reasonable request. All participants had signed informed consents.

\section{Conflicts of Interest}

The authors declare no conflict of interest.

\section{Reference}

Alexander's Disease. doi:10.1016/B978-0-12-385157-4.00087-7

Balbi, P., Salvini, S., Fundaro, C., Frazzitta, G., Maestri, R., Mosah, D., . . . Sechi, G. (2010). The clinical spectrum of late-onset Alexander disease: a systematic literature review. J Neurol, 257 (12), 1955-1962. doi:10.1007/s00415-010-5706-1

Brenner, M., Johnson, A. B., Boespflug-Tanguy, O., Rodriguez, D., Goldman, J. E., \& Messing, A. (2001). Mutations in GFAP, encoding glial fibrillary acidic protein, are associated with Alexander disease.Nat Genet, 27 (1), 117-120. doi:10.1038/83679

Bresciani, A., Spiezia, M. C., Boggio, R., Cariulo, C., Nordheim, A., Altobelli, R., . . . Weiss, A. (2018). Quantifying autophagy using novel LC3B and p62 TR-FRET assays. PLoS One, 13 (3), e0194423. doi:10.1371/journal.pone.0194423

Calabrese, M., Magliozzi, R., Ciccarelli, O., Geurts, J. J., Reynolds, R., \& Martin, R. (2015). Exploring the origins of grey matter damage in multiple sclerosis. Nat Rev Neurosci, 16 (3), 147-158. doi:10.1038/nrn3900

Chen, Y. S., Lim, S. C., Chen, M. H., Quinlan, R. A., \& Perng, M. D. (2011). Alexander disease causing mutations in the C-terminal domain of GFAP are deleterious both to assembly and network formation with the potential to both activate caspase 3 and decrease cell viability.Exp Cell Res, 317 (16), 2252-2266. doi:10.1016/j.yexcr.2011.06.017

Eijlers, A. J., Meijer, K. A., Wassenaar, T. M., Steenwijk, M. D., Uitdehaag, B. M., Barkhof, F., . . - Schoonheim, M. M. (2017). Increased default-mode network centrality in cognitively impaired multiple sclerosis patients. Neurology, 88 (10), 952-960. doi:10.1212/wnl.0000000000003689

Eshaghi, A., Marinescu, R. V., Young, A. L., Firth, N. C., Prados, F., Jorge Cardoso, M., . . . Ciccarelli, O. (2018). Progression of regional grey matter atrophy in multiple sclerosis. Brain, 141 (6), 1665-1677. doi:10.1093/brain/awy088

Eshaghi, A., Prados, F., Brownlee, W. J., Altmann, D. R., Tur, C., Cardoso, M. J., . . . group, M. s. (2018). Deep gray matter volume loss drives disability worsening in multiple sclerosis. Ann Neurol, 83 (2), 210-222. doi:10.1002/ana.25145 
Hol, E. M., \& Capetanaki, Y. (2017). Type III Intermediate Filaments Desmin, Glial Fibrillary Acidic Protein (GFAP), Vimentin, and Peripherin. Cold Spring Harbor Perspectives in Biology, 9 (12), a021642. doi:10.1101/cshperspect.a021642

Hsiao, V. C., Tian, R., Long, H., Der Perng, M., Brenner, M., Quinlan, R. A., \& Goldman, J. E. (2005). Alexander-disease mutation of GFAP causes filament disorganization and decreased solubility of GFAP. $J$ Cell Sci, 118 (Pt 9), 2057-2065. doi:10.1242/jcs.02339

Jones, J. R., Kong, L., Hanna, M. G. t., Hoffman, B., Krencik, R., Bradley, R., . . . Zhang, S. C. (2018). Mutations in GFAP Disrupt the Distribution and Function of Organelles in Human Astrocytes. Cell Rep, 25 (4), 947-958 e944. doi:10.1016/j.celrep.2018.09.083

Kwon, Y. T., \& Ciechanover, A. (2017). The Ubiquitin Code in the Ubiquitin-Proteasome System and Autophagy. Trends Biochem Sci, 42 (11), 873-886. doi:10.1016/j.tibs.2017.09.002

Li, R., Johnson, A. B., Salomons, G., Goldman, J. E., Naidu, S., Quinlan, R., . . . Brenner, M. (2005). Glial fibrillary acidic protein mutations in infantile, juvenile, and adult forms of Alexander disease.Ann Neurol, 57 (3), 310-326. doi:10.1002/ana.20406

Messing, A. (2018). Alexander disease. Handb Clin Neurol, 148 , 693-700. doi:10.1016/B978-0-444-64076$5.00044-2$

Middeldorp, J., Kamphuis, W., Sluijs, J. A., Achoui, D., Leenaars, C. H., Feenstra, M. G., . . Hol, E. M. (2009). Intermediate filament transcription in astrocytes is repressed by proteasome inhibition.FASEB J, 23 (8), 2710-2726. doi:10.1096/fj.08-127696

Minagar, A., Barnett, M. H., Benedict, R. H., Pelletier, D., Pirko, I., Sahraian, M. A., . . . Zivadinov, R. (2013). The thalamus and multiple sclerosis: modern views on pathologic, imaging, and clinical aspects.Neurology, 80 (2), 210-219. doi:10.1212/WNL.0b013e31827b910b

Perng, M. D., Su, M., Wen, S. F., Li, R., Gibbon, T., Prescott, A. R., . . . Quinlan, R. A. (2006). The Alexander Disease-Causing Glial Fibrillary Acidic Protein Mutant, R416W, Accumulates into Rosenthal Fibers by a Pathway That Involves Filament Aggregation and the Association of $\alpha$ B-Crystallin and HSP27. 79 (2), 197-213. doi:10.1086/504411

Prust, M., Wang, J., Morizono, H., Messing, A., Brenner, M., Gordon, E., . . . Vanderver, A. (2011). GFAP mutations, age at onset, and clinical subtypes in Alexander disease. Neurology, 77 (13), 1287-1294. doi:10.1212/wnl.0b013e3182309f72

Roosendaal, S. D., Bendfeldt, K., Vrenken, H., Polman, C. H., Borgwardt, S., Radue, E. W., . . . Geurts, J. J. (2011). Grey matter volume in a large cohort of MS patients: relation to MRI parameters and disability. Mult Scler, 17 (9), 1098-1106. doi:10.1177/1352458511404916

Sawaishi, Y. (2009). Review of Alexander disease: beyond the classical concept of leukodystrophy. Brain Dev, 31 (7), 493-498. doi:10.1016/j.braindev.2009.03.006

Tang, G., Perng, M. D., Wilk, S., Quinlan, R., \& Goldman, J. E. (2010). Oligomers of mutant glial fibrillary acidic protein (GFAP) Inhibit the proteasome system in alexander disease astrocytes, and the small heat shock protein alphaB-crystallin reverses the inhibition. J Biol Chem, 285 (14), 10527-10537. doi:10.1074/jbc.M109.067975

Tang, G., Xu, Z., \& Goldman, J. E. (2006). Synergistic effects of the SAPK/JNK and the proteasome pathway on glial fibrillary acidic protein (GFAP) accumulation in Alexander disease. J Biol Chem, 281 (50), 38634-38643. doi:10.1074/jbc.M604942200

Tang, G., Yue, Z., Talloczy, Z., Hagemann, T., Cho, W., Messing, A., . . . Goldman, J. E. (2008). Autophagy induced by Alexander disease-mutant GFAP accumulation is regulated by p38/MAPK and mTOR signaling pathways. Hum Mol Genet, 17 (11), 1540-1555. doi:10.1093/hmg/ddn042 
van der Knaap, M. S., Ramesh, V., Schiffmann, R., Blaser, S., Kyllerman, M., Gholkar, A., . . . Salomons, G. S. (2006). Alexander disease: ventricular garlands and abnormalities of the medulla and spinal cord.Neurology, 66 (4), 494-498. doi:10.1212/01.wnl.0000198770.80743.37

Widjaja, E., Zamyadi, M., Raybaud, C., Snead, O. C., \& Smith, M. L. (2013). Abnormal functional network connectivity among resting-state networks in children with frontal lobe epilepsy. AJNR Am J Neuroradiol, 34 (12), 2386-2392. doi:10.3174/ajnr.A3608

Yan, C. G., Wang, X. D., Zuo, X. N., \& Zang, Y. F. (2016). DPABI: Data Processing \& Analysis for (Resting-State) Brain Imaging.Neuroinformatics, 14 (3), 339-351. doi:10.1007/s12021-016-9299-4

Yang, H., Long, X. Y., Yang, Y., Yan, H., Zhu, C. Z., Zhou, X. P., . . . Gong, Q. Y. (2007). Amplitude of low frequency fluctuation within visual areas revealed by resting-state functional MRI. Neuroimage, 36 (1), 144-152. doi:10.1016/j.neuroimage.2007.01.054

Yoshida, T., Sasaki, M., Yoshida, M., Namekawa, M., Okamoto, Y., Tsujino, S., . . . Alexander Disease Study Group in, J. (2011). Nationwide survey of Alexander disease in Japan and proposed new guidelines for diagnosis. J Neurol, 258 (11), 1998-2008. doi:10.1007/s00415-011-6056-3

Yoshida, T., Sasayama, H., \& Nakagawa, M. (2009). The process of inducing GFAP aggregates in astrocytoma-derived cells is different between R239C and R416W mutant GFAP. A time-lapse recording study.Neurosci Lett, 458 (1), 11-14. doi:10.1016/j.neulet.2009.04.032

Zang, Y., Jiang, T., Lu, Y., He, Y., \& Tian, L. (2004). Regional homogeneity approach to fMRI data analysis. Neuroimage, 22 (1), 394-400. doi:10.1016/j.neuroimage.2003.12.030

\section{Figure legend}

Fig.1 Genetic and radiologic characteristics of patients who carry de novo variant in GFAP . (A-B) Chest radiography of patient P3433. Scoliosis was corrected after the operation. (C-D) Sequence chromatograms of GFAP gene of two probands, patient P4288 and patient P3433. (E) Patient P3433 demonstrated hyperintensity in the bilateral corona radiata, centrum semiovale and regions surrounding the 4 th ventricle in T2-weighted FLAIR images. (F) In the proband P4288, minor hyperintensity was observed while the distribution of these lesions was similar to patient P3433. (G) Patient P3433 presented atrophy in subcortical grey matter: bilateral putamen, thalamus and cerebellum, as well as atrophic white matter in corona radiata, centrum semiovale, cerebellopontine angle and medulla. $(\mathrm{H})$ Patient $\mathrm{P} 4288$ presented insignificant atrophy in grey matter, while the distribution of atrophic white matter was similar to patient P3433. Red/orange: the significantly larger volume of grey matter in normal controls than the patient. Yellow: the significantly larger volume of white matter in normal controls than the patient. (I) Patient P3433 presented higher ALFF in cerebellar vermis, cerebellopontine angles, occipital and posterior parietal cortex. Increased DC was observed in frontal and posterior cortex, overlapping with Reho mainly in cerebellum and posterior cortex. (J) Patient P4288 presented higher ALFF, DC and Reho in similar regions with patient P3433. Increased DC was additionally observed in bilateral insula. Red/orange: voxels with significantly higher centrality; Blue: voxels with significantly higher amplitude of low frequency fluctuation. Pink: voxels with significantly higher amplitude of regional homogeneity.

Fig .2Functional analysis of GFAP variants identified in this study. (A) The relative protein levels of GFAP$\mathrm{WT} /$ Mut in soluble fraction and insoluble fraction were detected by western blot (One-way ANOVA; $\mathrm{n}=3$ separate experiments; $\mathrm{ns}=$ non-significance, ${ }^{*} 0.01$ [?] $\mathrm{P}<0.05$, ${ }^{* * *} \mathrm{P}<0.001$ ). (B) Immunofluorescence showed distributions of GFAP-WT were diffuse with few aggregates, while mutant groups (p.E72K and p.T412I) and positive control (p.R239C) were presented as punctate inclusions in perinuclear areas. Scale bar, $10 \mu \mathrm{m}$. (C) After MG132 treatment, the soluble protein levels of 20S remained constant levels among groups while GFAP levels showed a decreased trend especially in WT, p.E72K and positive control groups (Two-way ANOVA; $\mathrm{n}=3$ separate experiments; ${ }^{*} \mathrm{P}<0.05$, ${ }^{*} \mathrm{P}<0.01$ ). (D-E) Immunofluorescence indicating that $20 \mathrm{~S}$ complex (red, D) and ubiquitin (red, E) in mutant groups and positive control exhibited as inclusions surrounding the nucleus, while diffuse distribution in cytoplasm was shown in WT group. 
Ubiquitin and 20S complexes were completely colocalized with mutant GFAP (green), respectively. Scale bar, $10 \mu \mathrm{m}$. (F) The soluble levels of GFAP and LC3-II in BafAI-treated group were increased, especially in mutant groups and positive control. (G) After BafAI treatment, the statistical analysis of GFAP levels was shown (Two-way ANOVA; $\mathrm{n}=3$ separate experiments; ns $=$ non-significance, $\left.{ }^{*} \mathrm{P}<0.05\right)$. (H-I) Immunostaining displayed that GFAP-Mut proteins (green) exhibited aggregates and nearly co-expressed LC3 (red) and LAMP1 (red). Scale bar, $10 \mu \mathrm{m}$.

Fig.3 Schematic diagram of GFAP structure with identified variants. Mutations identified in our study are in red font.

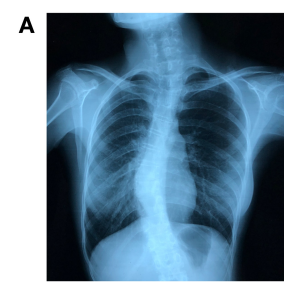

B
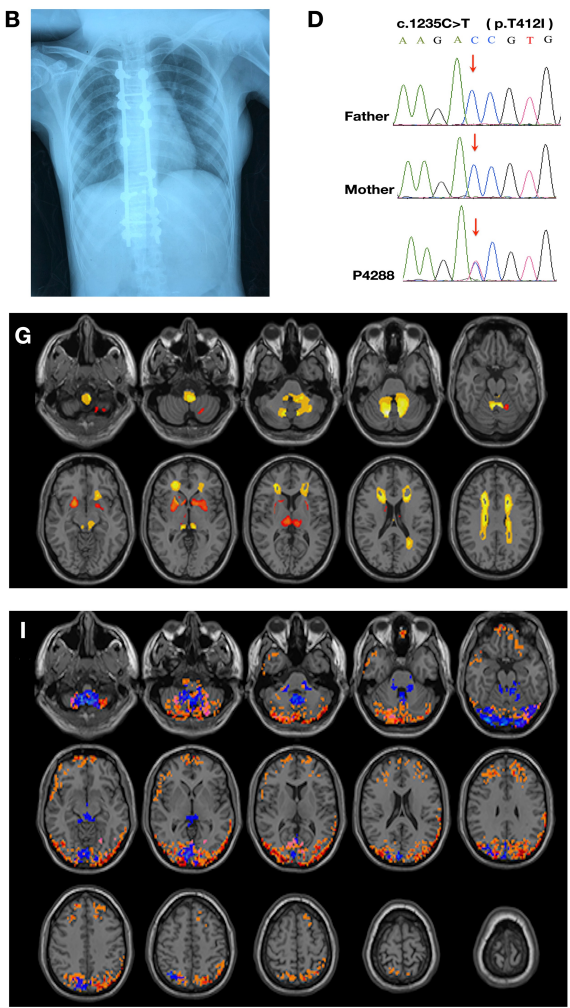

C

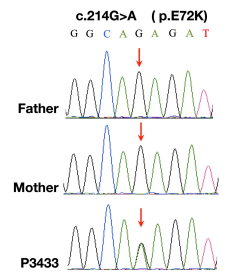

D

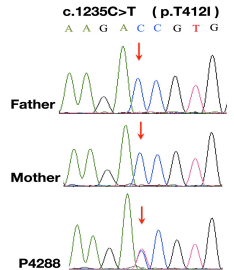

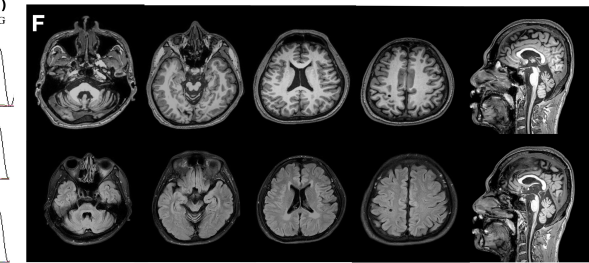
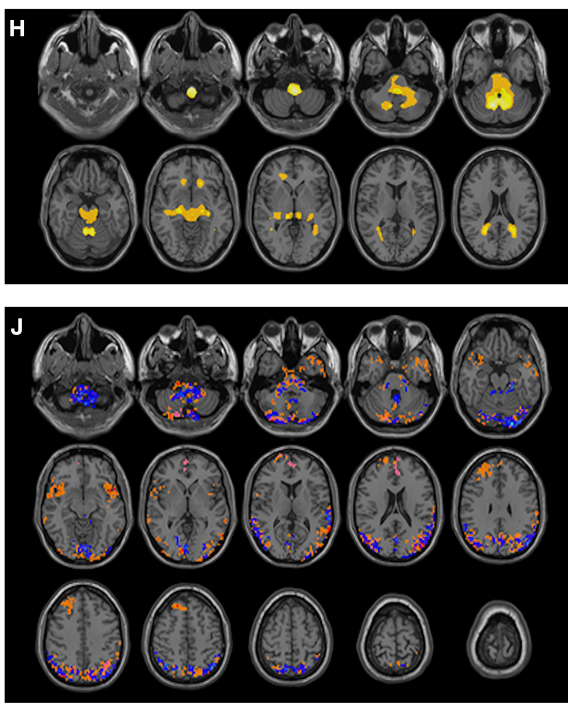
A

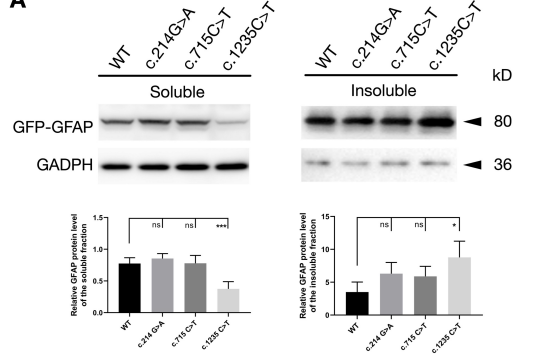

B
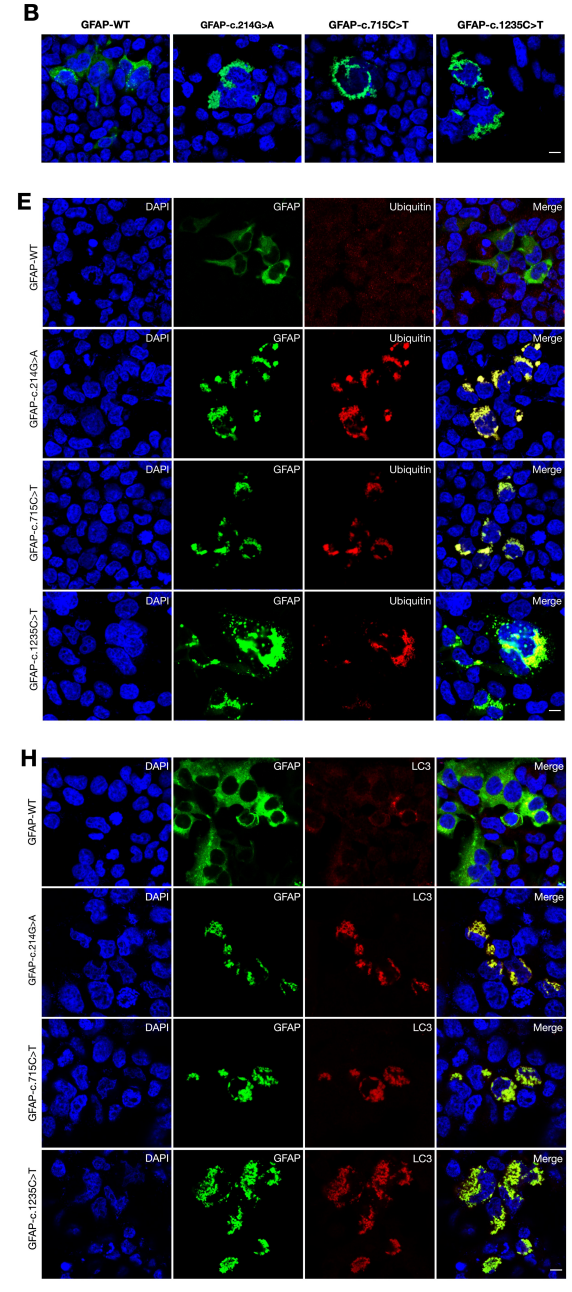

C
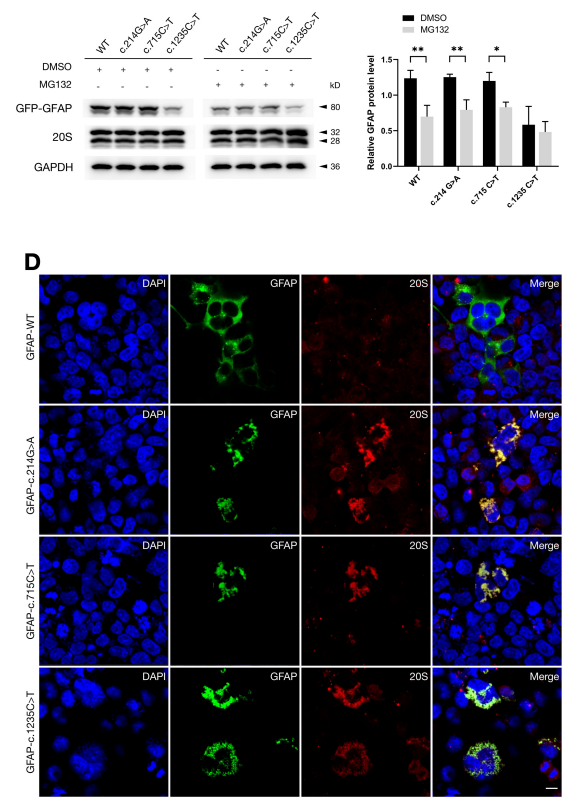

F $\quad$ (i)
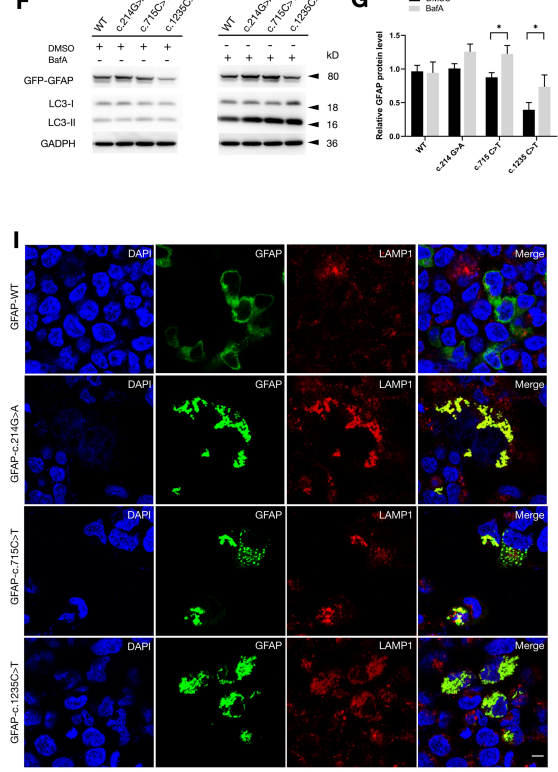


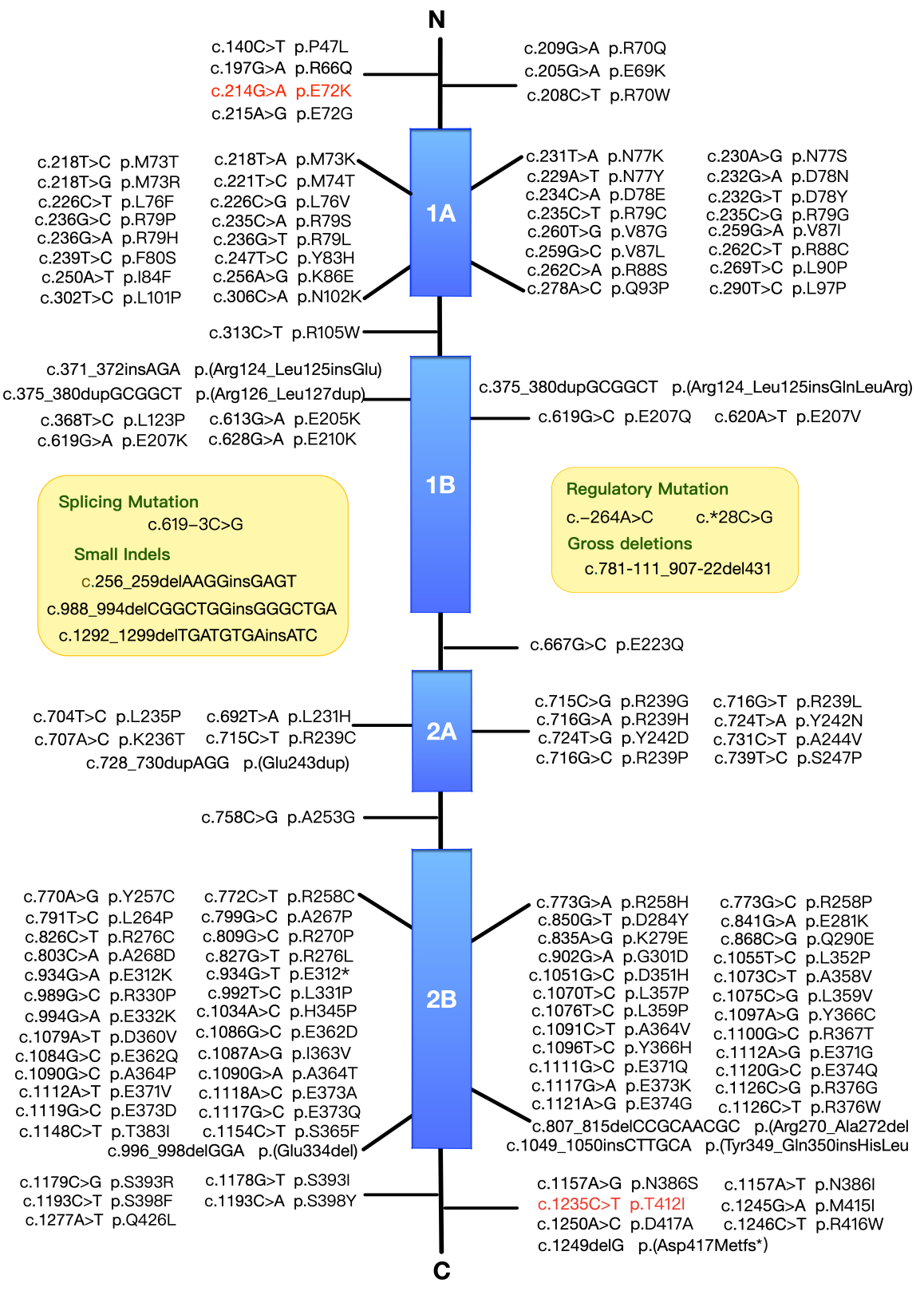

